\title{
Desenvolvimento reprodutivo de tourinhos Gir selecionados para produção de leite
}

\author{
[Reproductive development of Gyr young bulls selected for \\ milk production] \\ J.A.M. Martins ${ }^{1}$, F.A. Souza ${ }^{1}$, M.B.D. Ferreira ${ }^{2}$, P.E.M. Gonçalves ${ }^{1}$, L.L. Emerick ${ }^{1}$, \\ J.C. Dias ${ }^{3}$, T.G. Leite ${ }^{1}$, D.S. Graça ${ }^{1}$, V.J. Andrade ${ }^{1}$, V.R. Vale Filho ${ }^{1}$ \\ ${ }^{1}$ Escola de Veterinária - Universidade Federal de Minas Gerais - Belo Horizonte, MG \\ Av. Antônio Carlos, 6627 - Caixa Postal 567 \\ 30123-970 - Belo Horizonte, MG \\ ${ }^{2}$ Empresa de pesquisa Agropecuária de Minas Gerais (EPAMIG) - Belo Horizonte, MG \\ ${ }^{3}$ Instituto Agronômico do Paraná (IAPAR) /Fundação Araucária
}

\begin{abstract}
RESUMO
O estudo visou identificar tourinhos Gir precoces e não precoces quanto à puberdade e avaliar diferenças durante seu desenvolvimento reprodutivo. Peso vivo e perímetro escrotal foram mensurados mensalmente junto com a coleta e a avaliação física e morfológica do sêmen de 16 animais, dos 13 aos 23 meses de idade. Animais precoces foram mais leves na pré-puberdade e apresentaram menores idades à puberdade e à maturidade sexual - 17,0 e 18,7 meses -, respectivamente, - em relação aos não precoces - 19,2 e 20,5 meses, respectivamente. A motilidade aumentou na pré-puberdade dois meses mais cedo nos animais precoces - 1,75\% a 18,4\% dos 14 aos 17 meses - em relação aos não precoces - 2,5\% a 12,4\% dos 15 aos 18 meses de idade. Registrou-se aumento mais cedo da concentração espermática em animais precoces, a qual foi maior - 660 milhões $/ \mathrm{mL}$ - aos 23 meses em relação aos animais não precoces -66.7 milhões/mL. As diferenças observadas no desenvolvimento dos dois grupos foram favoráveis aos animais precoces e indicam que a seleção para a maturidade sexual precoce é indicada para a antecipação da fase reprodutiva de touros Gir.
\end{abstract}

Palavras-chave: avaliação de sêmen, bovinos, precocidade, puberdade, zebu

\begin{abstract}
This study aimed to identify precocious and non-precocious Gyr young bulls according to puberty and evaluate differences during their reproductive development. Live weight and scrotal circumference were measured monthly with collection and evaluation of semen samples from 16 animals, from 13 to 23 months of age. Precocious animals were lighter at the pre-puberty period and younger at puberty and sexual maturity, 17.0 and 18.7 months, respectively, regarding non-precocious, 19.2 and 20.5 months, respectively. Sperm motility increased during pre-puberty two months earlier for precocious animals, $1.75 \%$ to $18.4 \%$ from 14 to 17 months, regarding non-precocious, $2.5 \%$ to $12.4 \%$ from 16 to 18 months. Sperm concentration increase occurred earlier in precocious animals, and was higher, 669 million/mL, at 23 months of age in relation to non-precocious animals, 66.7 million/mL. The differences in reproductive development of both groups were favorable for precocious animals and indicate that the selection for precocious sexual maturity is advised to anticipate the reproductive phase of Gyr bulls.
\end{abstract}

Keywords: bovine, precocity, puberty, semen evaluation, zebu

Recebido em 17 de maio de 2010

Aceito em 27 de junho de 2011

E-mail: jammvet@gmail.com 


\section{INTRODUÇÃO}

Os bovinos zebuínos, por serem rústicos, são produtivos em climas tropicais mesmo consumindo forragem de baixa qualidade, tendo, no entanto, desenvolvimento sexual mais tardio que os animais de raças europeias (Nogueira, 2004).

A seleção de touros para precocidade sexual deve ser objetivo de produtores e pesquisadores, pois permite a introdução precoce de animais no manejo reprodutivo, aumentando sua vida útil e reduzindo o intervalo de gerações (Andréa et al., 2000). Eler et al. (2002), ao estudarem os critérios de seleção ligados à precocidade sexual, especialmente a probabilidade de prenhez aos 14 mese de idade, em uma população de 25.000 novilhas da raça Nelore, verificaram alta herdabilidade $\left(\mathrm{h}^{2}=0,67\right)$ para esta característica, indicando que filhas de determinados touros atingem a puberdade mais cedo do que filhas de outros touros. Tais resultados sugerem que a seleção de touros para a precocidade pode resultar em menor idade ao primeiro parto de suas filhas. A avaliação clínico-andrológica, visando à precocidade de touros de raças zebuínas a partir de um ano de idade, permite identificar reprodutores superiores cada vez mais jovens (Andrade et al., 2004; Vale Filho et al., 2004), sendo essencial para se acelerar o ganho genético por geração (Fordyce et al., 2002). Assim, o objetivo deste trabalho foi identificar tourinhos precoces e não precoces, bem como avaliar as diferenças no seu desenvolvimento reprodutivo até a maturidade sexual.

\section{MATERIAL E MÉTODOS}

Todas as técnicas e os procedimentos utilizados no presente estudo foram aprovados pelo Comitê de Ética em Experimentação Animal da Universidade Federal de Minas Gerais (protocolo no $55 / 2008$ )

Trinta tourinhos Gir-leiteiro, clinicamente normais, oriundos de linhagens com alta pressão de seleção para a produção de leite nas matrizes, de cinco propriedades no estado de Minas Gerais, foram acompanhados clínica e andrologicamente dos 13 aos 23 meses de idade. Os animais, submetidos a um período de adaptação de 30 dias antes do início do experimento, foram mantidos em confinamento individual e alimentados com dieta contendo $20 \%$ de proteína bruta (PB), $70 \%$ de nutrientes digestíveis totais (NDT) e $1,105 \mathrm{Mcal} / \mathrm{kg}$ de energia líquida, à base de $1 \%$ do peso vivo, durante todo o período experimental, e silagem de milho como volumoso. Sal mineral e água foram fornecidos ad libitum.

Avaliaram-se, mensalmente, o peso vivo (PV) e o perímetro escrotal (PE), e foram realizadas as coletas de sêmen. O PV foi obtido em balança e o PE foi mensurado com fita milimétrica no seu local de maior largura, envolvendo as duas gônadas. Avaliações do volume do ejaculado (VE), da concentração (CONC) e motilidade (MOT) espermáticas e do vigor (VIG) espermático, bem como dos percentuais de defeitos espermáticos maiores (DM), menores (Dm), totais (DT) e de espermatozoides normais (NORM), foram realizadas segundo as normas do Colégio Brasileiro de Reprodução Animal (Manual..., 1998).

Os dados foram ajustados de acordo com a idade à puberdade, segundo Brito et al. (2004). A puberdade em machos bovinos é definida como a idade em que o animal apresenta ejaculados com, no mínimo, 50 milhões de espermatozoides totais e $10 \%$ de motilidade (Wolf et al., 1965). Com base nesta definição, a idade em que os animais iniciaram a puberdade foi denominada momento 0 (zero dias em relação à puberdade) e foi adotado o máximo de observações existentes antes e após tal período, para verificar o desenvolvimento das características avaliadas. Doze animais entraram na puberdade antes do início do experimento, portanto sem observações pré-puberdade, e dois ainda não tinham atingido a puberdade no final do período experimental. Assim, dos 30 animais avaliados, apenas 16 continham informações completas 60 dias antes e 60 dias após o momento 0 . Sob esta estrutura de ajuste dos dados, todas as variáveis foram comparadas entre os grupos de animais precoces e não precoces - nas respectivas idades em relação à puberdade - -60, -30, 0, +30 e +60 dias.

Após o ajuste dos dados de acordo com a idade em relação à puberdade (dias), no momento 0 foi realizada distribuição de frequência das idades dos 16 animais estudados pelo procedimento FREQ do SAS (Statistical..., 2002), tendo como 
ponto de separação dos grupos a mediana -18 meses - das idades naquele período. Assim, animais com idades à puberdade abaixo da mediana foram considerados precoces (PREC), e com idades acima da mediana não precoces (NPREC), definição esta mais apropriada que animais tardios, pois todos os 16 animais estudados, mesmo aqueles que atingiram a puberdade com idades acima da mediana naquele período, atingiram a maturidade sexual - no mínimo $70 \%$ de espermatozoides normais com menos de $15 \%$ de defeitos espermáticos maiores, segundo Hopkins e Spitzer (1997), até 24 meses -, idade normalmente observada para a raça estudada.

Todas as variáveis foram submetidas aos testes de normalidade de Shapiro-Wilk e de assimetria e curtose, por meio do procedimento UNIVARIATE com a opção NORMAL do SAS (SAS, 2002). As variáveis VE e CONC foram transformadas logaritmicamente $(\log (\mathrm{x}+1))$, e as variáveis DM, Dm, DT e NORM sofreram transformação angular $(\operatorname{arco}-\operatorname{seno} \sqrt{(x / 100)})$ para a aproximação da distribuição normal. As variáveis MOT e VIG, por serem escores subjetivos de avaliação, foram consideradas variáveis não paramétricas (Sampaio, 2002).

As comparações das médias de idade, PV, PE, CONC, MOT, VIG, DM, Dm, DT e NORM foram realizadas, na puberdade e na maturidade sexual, por meio do teste de Fisher - para as variáveis paramétricas - e do teste de MannWhitney - para as variáveis não paramétricas -, entre os grupos de animais precoces e não precoces. As análises das variáveis foram realizadas sob delineamento em blocos ao acaso, em esquema de subparcelas, com oito repetições, sendo os grupos as parcelas, e as idades as subparcelas, por meio do procedimento MIXED do SAS (Littell et al., 1998). O efeito de grupo foi obtido pelo teste $\mathrm{F}$ e os efeitos de idade foram estimados por meio de ajustamento de modelos de regressão linear e quadrática com o uso do PROC REG do SAS. Nos casos em que a interação grupo versus idade foi significativa, ajustou-se um modelo de regressão para cada grupo. As comparações entre as idades dentro de cada grupo foram realizadas pelo teste SNK, por meio do procedimento GLM do SAS. As variáveis MOT e VIG foram comparadas pelo teste não paramétrico de Friedman (Sampaio, 2002). O ordenamento das observações foi realizado pelo PROC RANK do SAS (Ipe, 1987), seguido pela análise dos ordenamentos por meio do PROC MIXED.

As correlações entre as variáveis paramétricas foram estimadas pelo coeficiente de correlação de Pearson por meio do PROC CORR, e as associações entre as variáveis paramétricas e não paramétricas pelo coeficiente de correlação de Spearman por meio do PROC CORR com a opção SPEARMAN do SAS (Statistical..., 2002).

\section{RESULTADOS E DISCUSSÃO}

Não houve diferenças entre grupos $(\mathrm{P}>0,05)$ para as características estudadas, exceto para a idade, tanto na puberdade como na maturidade sexual $(\mathrm{P}<0,01)$ (Tab. 1), indicando que os animais precoces na puberdade também o são na maturidade sexual. Em média, os animais precoces atingiram a puberdade dois meses mais cedo do que os não precoces.

As idades à puberdade e à maturidade sexual foram mais baixas que as descritas por Brito et al. (2004), ao registrarem idades de 20 e 23,8 meses, para puberdade e maturidade na raça Nelore, respectivamente. A diferença entre os dois estudos reflete as diferenças de manejo alimentar dos experimentos. No presente experimento, os animais foram mantidos em sistema intensivo e alimentados com dieta $(1 \%$ PV) de elevado conteúdo proteico, além de silagem de milho como volumoso. Já no estudo de Brito et al. (2004), foram manejados extensivamente do nascimento até o final do experimento, em pasto de Brachiaria brizantha, apenas com suplementação mineral ad libitum.

Barth et al. (2008) avaliaram o efeito de três planos nutricionais distintos - baixo, médio e alto contento $0 \%, 6,6 \%$ e $37 \%$ de concentrado, respectivamente - sobre o desenvolvimento sexual de touros europeus e verificaram que os animais suplementados com alto plano nutricional atingiram a idade à puberdade mais cedo do que os animais suplementados com os planos médios e baixos. Assim, verifica-se a influência do plano alimentar sobre a idade à puberdade em bovinos. 
Tabela 1. Médias e desvios-padrão de idade, peso vivo, perímetro escrotal e características seminais à puberdade $^{1}$ e à maturidade sexual ${ }^{2}$ em tourinhos Gir-leiteiro precoces e não precoces

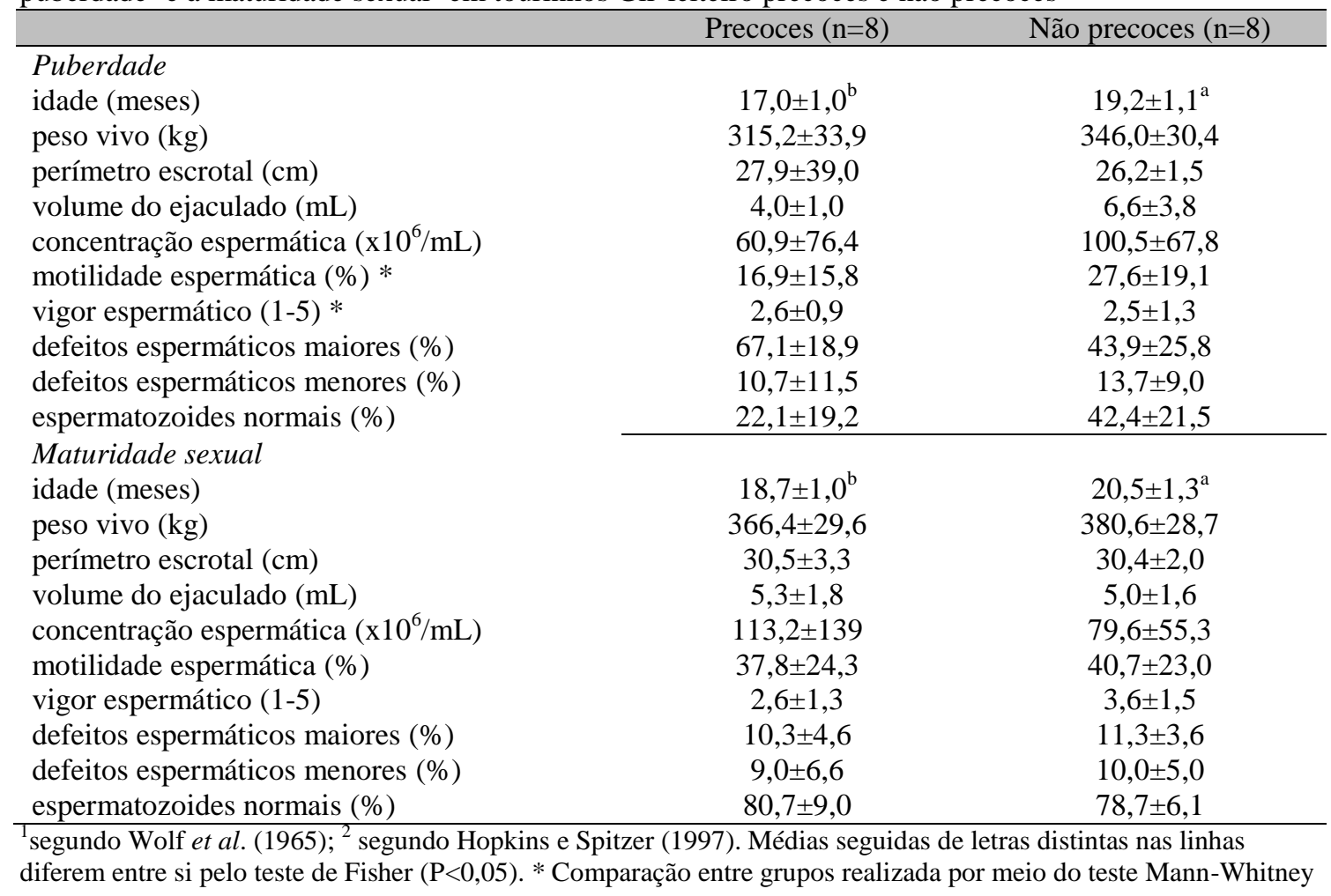

Em ambos os grupos, o período de maior ganho de peso foi observado entre 15 e 21 meses de idade. Não houve efeito de grupo e da interação grupo versus idade sobre o PV, demonstrando que o desenvolvimento segundo a idade foi igual para animais precoces e não precoces. No entanto, a mesma característica avaliada segundo a idade em relação à puberdade mostrou efeito de idade, de grupo, bem como a interação grupo versus idade (Fig. 1B). Os animais precoces foram mais leves aos 60 e aos 30 dias antes da puberdade, no entanto, a partir do momento zero até 60 dias após a puberdade, o incremento no peso vivo dos animais de ambos os grupos foi semelhante. Este resultado sugere que os animais NPREC apresentaram maior desenvolvimento corporal no período pré-puberal para iniciar a puberdade.

O peso vivo apresentou padrão de desenvolvimento quadrático em função da idade (Fig. 1A): $y=-461,43+65,27 x-1,2 x^{2}, \mathrm{R}^{2}$ $=0,73$. O padrão difere do descrito por TorresJúnior e Henry (2005), que encontraram crescimento linear do peso em função da idade, dos 7,5 aos 30,7 meses de idade, em touros Guzerá. Contudo, quando se avaliou o PV em função da idade à puberdade, verificou-se efeito linear dos 60 dias antes aos 60 dias depois da puberdade. Como o efeito da interação de grupo versus idade foi significativo, um modelo de regressão linear foi ajustado para cada grupo. Os animais precoces, apesar de serem mais leves na pré-puberdade, ganharam mais peso em relação aos não precoces, de acordo com os coeficientes angular da regressão dos seus respectivos modelos. O ganho de peso dos animais precoces (Fig. 1B) está representado pelo modelo $y=218,03+30,32 x, \mathrm{R}^{2}=0,67$, indicando que o ganho médio mensal foi de $30,32 \mathrm{~kg}$. Já o ganho de peso dos animais não precoces (Fig. 1B) está representado pelo modelo $y=282,87+19,85 x, \mathrm{R}^{2}=0,47$, indicando que o ganho médio mensal foi de apenas $19,85 \mathrm{~kg}$. Tais resultados explicam a inclinação mais acentuada do modelo que representa o ganho de peso dos animais precoces em relação aos não precoces. 

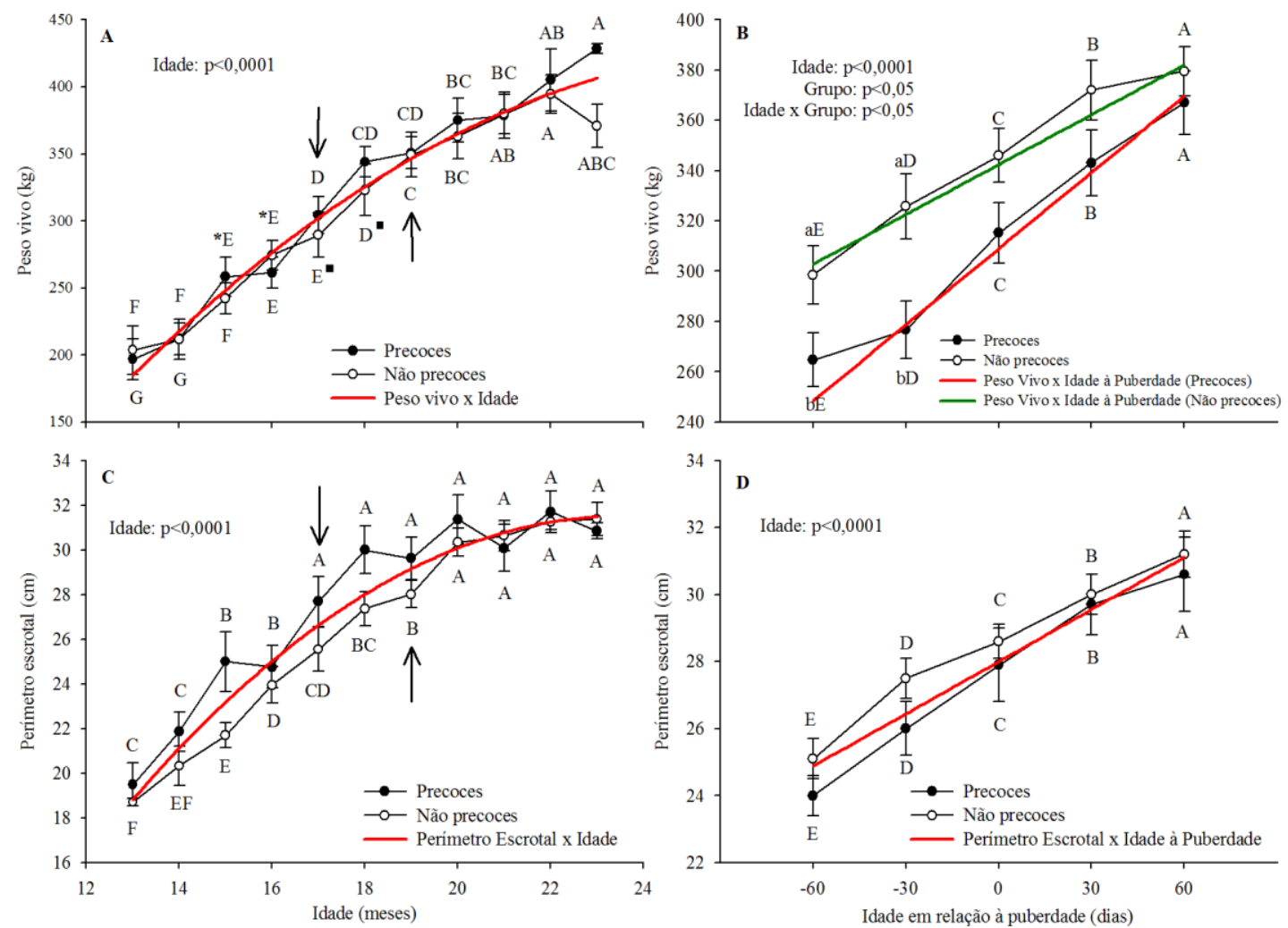

Figura 1. Médias ( \pm erros-padrão) do peso vivo (PV) e do perímetro escrotal (PE) segundo a idade (A e C) e a idade em relação à puberdade (B e D) em tourinhos Gir precoces e não precoces. Setas indicam idade média à puberdade ${ }^{1} \mathrm{em}$ tourinhos precoces $(\downarrow)$ e não precoces $(\uparrow)$. As linhas vermelhas em A e C representam os modelos de regressão quadrática para peso vivo $(\mathrm{A})$ e perímetro escrotal $(\mathrm{B})$ em relação à idade, e em $\mathrm{D}$ em relação à idade à puberdade. Em B, as linhas vermelha e verde representam os modelos de regressão linear do PV em função da idade à puberdade para animais precoces e não precoces, respectivamente. Médias sobrescritas com letras minúsculas distintas diferem entre grupos dentro de idade $(\mathrm{A} \mathrm{e} \mathrm{C})$ e idade em relação à puberdade $(\mathrm{B}$ e $\mathrm{D})$ pelo teste $\mathrm{F}(\mathrm{P}<0,05)$. Médias sobrescritas com letras maiúsculas distintas diferem entre as idades dentro de cada grupo pelo teste SNK $(\mathrm{P}<0,05) .{ }^{1}$ Segundo Wolf et al. $(1965)$

O perímetro escrotal variou de 19,5 a $30,8 \mathrm{~cm}$ e de 18,7 a $31,4 \mathrm{~cm}$, dos 13 aos 23 meses de idade para animais PREC e NPREC, respectivamente, com efeito significativo apenas da idade (Fig. 1C) e da idade em relação à puberdade (Fig. 1D) sobre o PE. $\mathrm{O}$ desenvolvimento dessa característica foi semelhante para os dois grupos, contudo, nos animais precoces, o tamanho dos testículos foi condizente tanto com a puberdade quanto com a maturidade sexual, que ocorreu dois meses mais cedo que a dos animais não precoces (Fig. 1C). O PE é uma medida amplamente usada na seleção de reprodutores por apresentar elevada herdabilidade (Ferraz e Eler, 2007) e pelas associações positivas com características produtivas e reprodutivas, como ganho de peso, qualidade do sêmen e precocidade sexual. Estudos demonstram que o
PE apresenta correlações positivas com peso corporal (Torres-Júnior e Henry, 2005), peso à desmama (Martins Filho e Lôbo, 1994), com motilidade espermática (Pastore et al., 2008), produção espermática diária (Palasz et al., 1994) e morfologia espermática (Silva et al., 2002). Apresenta, ainda, correlação genética negativa, porém favorável, com idade ao primeiro parto e com intervalo entre partos em novilha meiasirmãs paternas de touros, de modo que as meiasirmãs de touros com maiores perímetros escrotais apresentaram menores idades ao primeiro parto e menores intervalos entre gerações, conforme estudo de Martins Filho e Lôbo (1994), na raça Nelore.

O melhor ajuste do desenvolvimento testicular em função da idade foi apresentado pelo modelo 
quadrático (Fig. 1C): $y=-31,67+5,37 x-0,11 x^{2}$, $\mathrm{R}^{2}=0,69$. Segundo Rawlings et al. (2008), o padrão de desenvolvimento testicular em touros é sigmoide, com crescimento lento no período infantil, seguido de rápido crescimento durante a fase puberal e estabilização ao atingir a maturidade sexual. Neste estudo, o modelo quadrático foi o que melhor representou o padrão de aumento no perímetro escrotal, uma vez que os dados foram obtidos somente a partir dos 13 meses de idade.

Observou-se efeito linear quando se avaliou o PE segundo a idade em relação à puberdade (Fig. 1D). Como não houve interação significativa de grupo versus idade em relação à puberdade, pode-se afirmar que o desenvolvimento do PE para ambos os grupos foi semelhante $\mathrm{e}$ representado pelo modelo $y=23,32+1,56 x$, $\mathrm{R}^{2}=0,53$. $\mathrm{O}$ aumento médio mensal no perímetro escrotal, dos 60 dias antes aos 60 dias depois da puberdade, foi de $1,56 \mathrm{~cm}$, como mostra o coeficiente angular da regressão.

Apenas o efeito de idade foi significativo sobre o volume do ejaculado (Fig. 2A). Os testículos, o epidídimo e, principalmente, as glândulas sexuais acessórias (GSA) são responsáveis pela produção e secreção do plasma seminal (Senger, 2003). Rawlings et al. (2008) verificaram rápido crescimento testicular e de estruturas sexuais acessórias dependentes de andrógenos após cinco meses de idade, durante o rápido aumento na secreção de testosterona nessa fase. Acredita-se, portanto, que o aumento do volume do ejaculado com a idade dos animais possa estar associado à ação daquele andrógeno sobre as estruturas responsáveis pela produção e secreção do plasma seminal.

Houve efeito de idade e de grupo sobre a concentração (Fig. 2B). Aos 16, 17 e 18 meses de idade, a concentração espermática foi maior no grupo de animais precoces, indicando que estes apresentam desempenho espermatogênico mais acentuado durante o desenvolvimento sexual. A concentração também foi maior aos 23 meses de idade, época em que os animais já se encontravam na maturidade sexual. Do ponto de vista econômico, este resultado sugere que animais precoces seriam altamente indicados para centrais de inseminação artificial, tanto por sua utilização mais precoce nos testes de progênie, quanto pela quantidade de doses de sêmen produzidas, aumentando a comercialização de material genético de alto valor e a velocidade da propagação gênica de interesse à melhoria de rebanhos.

A concentração espermática apresentou desenvolvimento quadrático em relação à idade (Fig. 2B). Como o efeito da interação de grupo versus idade não foi significativo, o aumento da concentração espermática foi semelhante para animais PREC e NPREC, justificando o ajuste de apenas um modelo (Fig. 2B): $y=-14,5+1,32 x-0,02 x^{2}, \quad \mathrm{R}^{2}=0,64$. Observa-se que o aumento da CONC em animais precoces iniciou-se mais cedo e foi mais acentuado do que nos animais não precoces. A CONC também apresentou resposta quadrática em relação ao PE segundo a equação: $y=-4,2+0,08 x+0,006 x^{2}, \quad \mathrm{R}^{2}=0,69 . \quad \mathrm{O}$ diâmetro dos túbulos seminíferos cresce gradativamente no período infantil e mais rapidamente no período puberal. Aumentos no diâmetro e no comprimento dos túbulos seminíferos influenciam diretamente o tamanho testicular (Rawlings et al., 2008) e resultam em maior produção espermática.

A idade também apresentou efeito significativo $(\mathrm{P}<0,05)$ sobre a motilidade espermática, com aumento mais acelerado entre 16 e 19 meses para animais PREC, e entre 18 e 20 meses de idade para animais NPREC, (Fig. 3A).

Avaliando-se a motilidade espermática segundo a idade em relação à puberdade, verifica-se que tais períodos corresponderam a -30 a +60 dias para animais PREC e -30 a +30 dias para animais NPREC (Fig. 3B), evidenciando aumento da motilidade espermática no período pré-puberal para ambos os grupos. Almquist e Barber (1974) tiveram sucesso em coletar os primeiros espermatozoides móveis de touros da raça Charolesa, criados em alto plano nutricional, às 39 semanas de idade, duas semanas antes do início da puberdade, que foi observada às 41 semanas. 

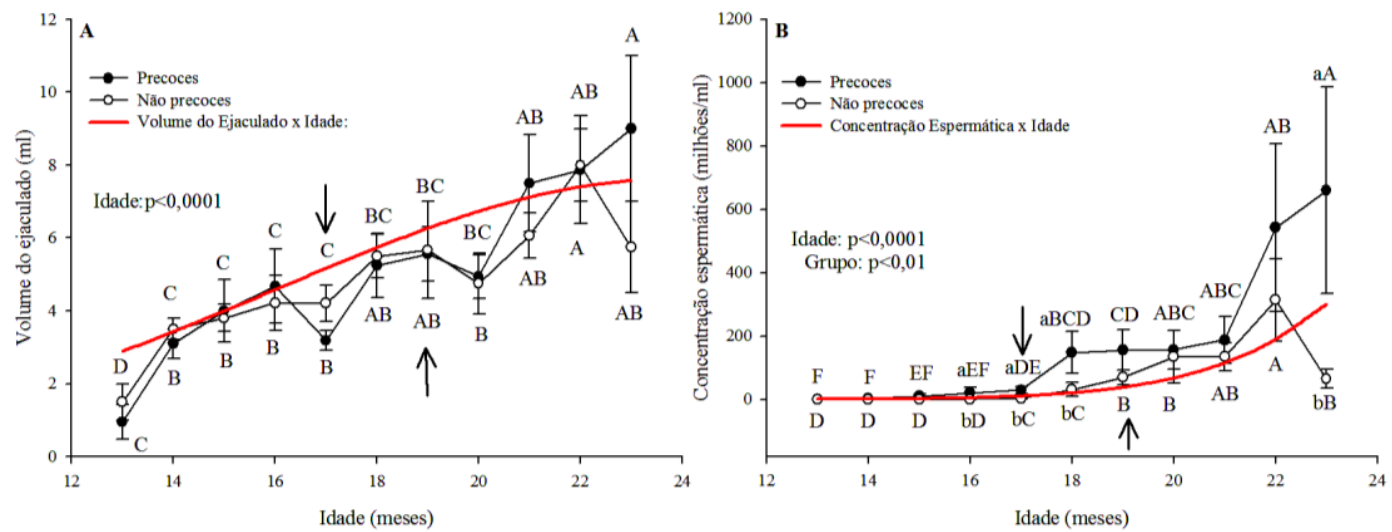

Figura 2. Médias ( \pm erros-padrão) do volume do ejaculado (VE - A) e da concentração espermática (CONC - B) segundo a idade em tourinhos Gir precoces e não precoces. Setas $(\downarrow \uparrow)$ indicam idade média à puberdade ${ }^{1}$ nos grupos precoces e não precoces, respectivamente. A linha vermelha representa o ajuste do modelo de regressão quadrática para VE (A) e para CONC (B) de ambos os grupos em relação à idade. Médias sobrescritas com letras minúsculas distintas diferem entre grupo dentro da idade pelo teste de Fisher $(\mathrm{F})(\mathrm{P}<0,05)$. Médias sobrescritas com letras maiúsculas distintas diferem entre idades dentro de cada grupo pelo teste $\mathrm{SNK}(\mathrm{P}<0,05)$.

${ }^{1}$ Segundo Wolf et al. (1965).
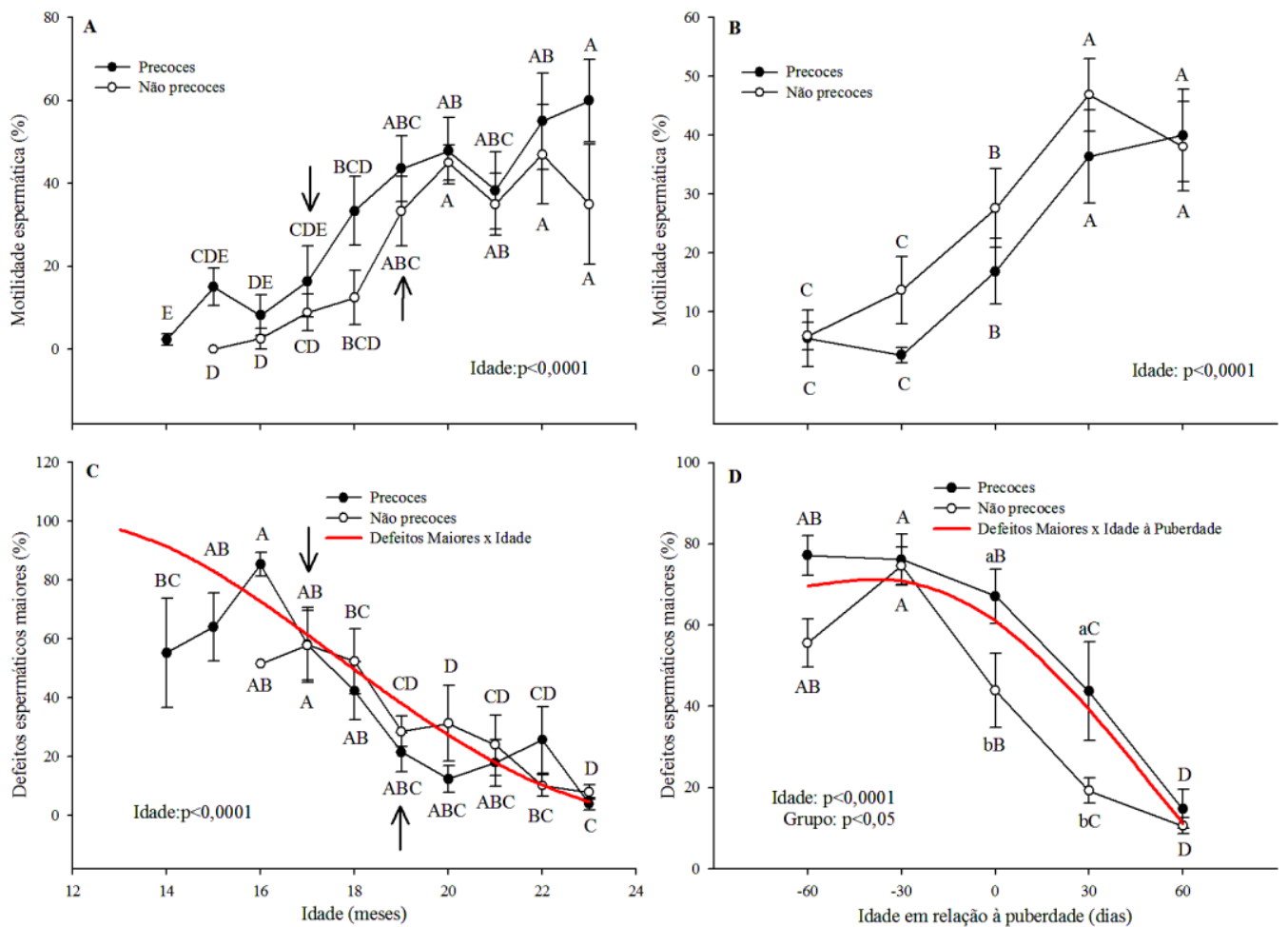

Figura 3. Médias ( \pm erros-padrão) da motilidade espermática (MOT) e dos defeitos espermáticos maiores (DM) segundo a idade (A e C) e a idade em relação à puberdade (B e D) em tourinhos Gir precoces e não precoces. Setas $(\downarrow \uparrow)$ indicam idade média à puberdade ${ }^{1}$ nos grupos precoces e não precoces, respectivamente. A linha vermelha representa o ajuste do modelo de regressão quadrática para DM de ambos os grupos em relação à idade e à idade à puberdade. Médias sobrescritas com letras minúsculas distintas diferem entre os grupos dentro da idade pelo teste $\mathrm{F}$ $(\mathrm{P}<0,05)$. Médias sobrescritas com letras maiúsculas distintas diferem entre idades dentro de cada grupo pelo teste SNK $(\mathrm{P}<0,05)$

${ }^{1}$ Segundo Wolf et al. (1965). 
Resultados similares foram registrados por Lunstra e Echternkamp (1982) em gado europeu, ao verificarem que o período de mais rápido aumento na motilidade espermática progressiva ocorreu entre duas semanas antes e seis semanas após a puberdade. Dois meses antes do início da puberdade, ocorre o início do aumento linear nas concentrações séricas de LH e de testosterona em Bos taurus taurus (Lunstra et al., 1978). Assim, o aumento da motilidade no período pré-puberal pode ser explicado pelo aumento na secreção de andrógenos que regulam as funções epididimárias, como a maturação espermática, e a secreção das glândulas sexuais acessórias no mesmo período.

Os defeitos espermáticos maiores diminuíram com a idade (Fig. 3C) em ambos os grupos, com maior declínio dos DM entre $16(85,4 \%)$ e 23 (4\%) meses e entre 17 e 23 meses de idade para os grupos PREC e NPREC, respectivamente. Schmidt-Hebbel et al. (2000) verificaram diminuição nos DM entre 14,2 e 25 meses de idade em touros Gir selecionados para reprodução. Verificou-se efeito de idade e de grupo sobre DM (Fig. 3D). Os animais não precoces apresentaram menor percentual de DM na puberdade (momento 0) e 30 dias depois. Entretanto, ambos os grupos apresentaram percentuais de defeitos maiores semelhantes
60 dias após a puberdade, o que sugere a possibilidade de iniciar a seleção dos reprodutores ainda mais cedo, visando não só à eficiência reprodutiva como também à precocidade.

Não houve efeito significativo da interação de grupo versus idade, indicando que o desenvolvimento dos defeitos espermáticos maiores foi semelhante para ambos os grupos e representado por modelos quadráticos tanto em relação à idade $-y=1,9-0,04 x-0,0014 x^{2}$, $\mathrm{R}^{2}=0,36$ - como à idade em relação à puberdade $-y=0,85+0,12 x-0,06 x^{2}, \quad \mathrm{R}^{2}=0,67$. Defeitos maiores foram registrados a partir dos 16 meses de idade (Fig. 3C) no grupo de animais não precoces, uma vez que não foram encontradas células espermáticas antes desta idade, possivelmente por ainda não ter sido iniciado o processo espermatogênico. Já os animais precoces apresentaram células espermáticas no ejaculado aos 13 meses de idade.

Os coeficientes de correlação de Pearson e de Spearman entre as características estudadas estão demonstrados na Tab. 2.

Tabela 2. Correlações de Pearson e Spearman entre os parâmetros estudados no desenvolvimento sexual de tourinhos Gir-leiteiro

\begin{tabular}{cccccccccc}
\hline & PE & PV & VE & CONC & MOT* & VIG $^{*}$ & DM & DT & NORM \\
\hline Idade & $0,78^{1}$ & $0,83^{1}$ & $0,55^{1}$ & $0,77^{1}$ & $0,57^{1}$ & $0,48^{1}$ & $-0,60^{1}$ & $-0,56^{1}$ & $0,56^{1}$ \\
PE & & $0,82^{1}$ & $0,54^{1}$ & $0,81^{1}$ & $0,43^{2}$ & $0,39^{2}$ & $-0,51^{1}$ & $-0,46^{1}$ & $0,46^{1}$ \\
PV & & & $0,58^{1}$ & $0,76^{1}$ & $0,47^{1}$ & $0,35^{2}$ & $-0,60^{1}$ & $-0,55^{1}$ & $0,55^{1}$ \\
VE & & & & $0,41^{1}$ & $0,04^{\mathrm{ns}}$ & $-0,08^{\mathrm{ns}}$ & $-0,31^{2}$ & $-0,28^{2}$ & $0,28^{2}$ \\
CONC & & & & & $0,52^{1}$ & $0,59^{1}$ & $-0,52^{1}$ & $-0,51^{1}$ & $0,51^{1}$ \\
MOT $^{*}$ & & & & & & $0,86^{1}$ & $-0,44^{2}$ & $-0,44^{2}$ & $0,44^{2}$ \\
VIG* & & & & & & $-0,30^{3}$ & $-0,28^{3}$ & $0,28^{3}$ \\
DM & & & & & & & $0,95^{1}$ & $-0,95^{1}$ \\
DT & & & & & & & & $-1,00^{1}$
\end{tabular}

PE: perímetro escrotal; PV: peso vivo; VE: volume do ejaculado; CONC: concentração espermática; MOT: motilidade espermática; VIG: vigor espermático; DM: defeitos espermáticos maiores; DT: defeitos espermáticos totais; NORM: espermatozoides normais.

${ }^{1} \mathrm{P}<0,0001$; ${ }^{2} \mathrm{P}<0,01 ;{ }^{3} \mathrm{P}<0,05$; ns: não significativo; * correlação de Spearman.

Com o avanço da idade, houve aumento significativo do PE, PV, VE, da CONC, MOT, VIG e NORM e diminuição de DM, Dm e totais DT, confirmando os resultados descritos anteriormente. O PE apresentou correlação significativa e de alta magnitude com o peso corporal, similar aos registrados por outros autores, razão pela qual é utilizado como ferramenta de seleção tanto para ganho de peso como para precocidade. Moura et al. (2002) 
verificaram que touros Nelores mais pesados aos 10 meses já apresentavam maiores PE $(r=0,62)$. Aos 30 meses, a correlação de Pearson entre o PE e o peso corporal foi significativa, porém de menor magnitude $(r=0,53)$.

O PE apresentou correlação significativa e de média magnitude com o VE e alta correlação com a CONC. Foi observada diminuição no percentual de DM com o aumento da idade, do peso e do PE, demonstrada pelas correlações negativas entre as variáveis. Foram observadas associações significativas de média magnitude entre idade, motilidade e vigor espermáticos. A idade foi mais marcante para o percentual de espermatozoides normais, DM e DT, sugerindo a evolução dos processos espermatogênico e de maturação espermática durante o desenvolvimento sexual dos touros.

As correlações entre PE, MOT, DM e DT foram semelhantes às descritas por Folhadella et al. (2006) - PE x MOT = 0,42 e PE x DM = -0,49. Corroborando, ainda, com aqueles autores, as correlações entre a morfologia espermática e a motilidade foram negativas, denotando a associação entre a morfologia do espermatozoide e sua motilidade.

\section{CONCLUSÕES}

Sob as mesmas condições de manejo nutricional intensivo, tourinhos Gir-leiteiro precoces à puberdade atingem a maturidade sexual dois meses mais cedo que animais não precoces. Uma vez que a seleção cada vez mais cedo de reprodutores de raças zebuínas com alto potencial genético e de reprodução é recomendada, a redução de dois meses na idade à puberdade e na maturidade pode ser significativa tanto do ponto de vista econômico como de melhoramento genético.

\section{AGRADECIMENTOS}

Às fazendas, Brasília, Calciolândia, Poções e São Bento, à Escola de Veterinária da Universidade Federal de Minas Gerais, à Empresa de Pesquisa Agropecuária de Minas Gerais (EPAMIG), à FAPEMIG e à CAPES, pelo apoio, parceria e financiamento.

\section{REFERÊNCIAS BIBLIOGRÁFICAS}

ALMQUIST, J.O.; BARBER, K.A. Puberal characteristics and early growth of Charolais bulls on high nutrient allowance. J. Anim. Sci., v.38, p.831834, 1974.

ANDRADE, V.J.; DIAS, J.C., SALVADOR, D.F. et al. Andrological characteristics of young (15-27 months old) Guzerat (Bos taurus indicus) bulls, raised on pasture in Minas Gerais, Brazil. In: INTERNATIONAL CONGRESS ON ANIMAL REPRODUCTION, 15., 2004, Porto Seguro. Anais... Belo Horizonte: CBRA, 2004. v.1, p.174.

ANDRÉA, M.V.; MARCONDES, C.R.; LÔBO, R.B. et al. Marcadores Moleculares Associados à Precocidade Sexual de Novilhas da Raça Nelore. In: SIMPÓSIO NACIONAL DE MELHORAMENTO ANIMAL, 3., 2000, Belo Horizonte. Anais... Belo Horizonte: FEP MVZ, 2000. v.1, p.427-429.

BARTH, A.D.; BRITO, L.F.C.; KASTELIC, J.P. The effect of nutrition on sexual development of bulls. Theriogenology, v.70, p.485-494, 2008.

BRITO, L.F.C.; SILVA, A.E.D.F.; UNANIAN, M.M. et al. Sexual development in early- and late-maturing Bos indicus and Bos indicus $x$ Bos taurus crossbred bulls in Brazil. Theriogenology, v.62, p.1198-1217, 2004.

ELER, J.P.; SILVA, J.A.V.; FERRAZ, J.B.S. et al. Genetic evaluation of the probability of pregnancy at 14 months for Nellore heifers. J. Anim. Sci., v.80, p.951-954, 2002.

FERRAZ, J.B.S.; ELER, J.P. Seleção de bos indicus para precocidade sexual. Rev. Bras. Reprod. Anim., v.31, p.167-171, 2007.

FOLHADELLA, I.M.; SÁ, W.F.; FERREIRA, A.M. et al. Características andrológicas de touros da raça Gir. Arq. Bras. Med. Vet. Zootec., v.58, p.809-815, 2006.

FORDYCE, G.; FITZPATRICK, L.A.; COOPER, N.J. et al. Bull selection and use in Northern Australia 5: Social behavior and management. Anim. Reprod. Sci., v.71, p.81-99, 2002.

HOPKINS F.M.; SPITZER, J.C. The new Society for Theriogenology breeding soundness evaluation system. Vet. Clin. N. Am.: Food. Anim. Pract., v.13, p.283-293, 1997.

IPE, D. Performing the Friedman test and the associated multiple comparison test using PROC GLM. In. SAS USERS GROUP INTERNATIONAL CONFERENCE. 20., 1987 Dallas, Texas, EUA. Proceedings..., SAS Institute 1987. v.12, p.1146-1148. 
LITTELL, R.C.; HENRY, P.R.; AMMERMAN, C.B. Statistical analysis of repeated measures data using SAS procedures. J. Anim. Sci., v.78, p.1216-1231, 1998.

LUNSTRA D.D.; ECHTERNKAMP, S.E. Puberty in beef bulls: acrosome morphology and semen quality in bulls of different breeds. J. Anim. Sci., v.55, p.638648. 1982.

LUNSTRA, D.D.; FORD, J.J.; ECHTERNKAMP, S.E. Puberty in beef bulls: hormone concentrations, growth, testicular development, sperm production and sexual aggressiveness in bulls of different breeds. $J$. Anim. Sci., v.46, p.1054-1062, 1978.

MANUAL para exame andrológico e avaliação de sêmen animal. 2.ed. Belo Horizonte: CBRA, 1998. $49 \mathrm{p}$.

MARTINS FILHO, R.; LÔBO, R.B. Correlações genéticas entre circunferência escrotal e características reprodutivas de fêmeas em bovinos da raça Nelore. Cienc. Agron., v.25, p.10-15, 1994.

MOURA, A.A.A.; RODRIGUES, G.R.; MARTINS FILHO, R. Desenvolvimento ponderal e testicular, concentrações periféricas de testosterona e características de abate em touros da raça Nelore. Rev. Bras. Zootec., v.31, supl.2, p.934-943, 2002.

NOGUEIRA, GP. Puberty in South American Bos indicus (Zebu) Cattle. Anim. Reprod. Sci., v.82-83, p.361-372, 2004.

PALASZ, A.T.; CATES, W.F.; BARTH, A.D. et al. The relationship between scrotal circumference and quantitative testicular traits in yearling beef bulls. Theriogenology, v.42, p.715-726, 1994.

PASTORE, A.A.; TONIOLLO, G.H.; LÔBO, R.B. et al. Características biométricas, testiculares, seminais e parâmetros genéticos de touros pertencentes ao programa de melhoramento genético da raça Nelore. Ars Vet., v.24, p.134-141, 2008.
RAWLINGS, N.; EVANS, A.C.O.; CHANDOLIA, R.K. et al. Sexual maturation in the bull. Reprod. Domest. Anim., v.43, Suppl. 2, p.295-301, 2008.

SAMPAIO, I.B.M. Estatística aplicada à experimentação animal. 2ed. Belo Horizonte, MG: FEPE MVZ, 2002. 265p.

Statistical analysis system: user's Guide-SAS, release. Cary, NC: SAS Institute, 2002.

SCHMIDT-HEBBEL, J.; TONIOLLO G.H.; LEITE, F.G. et al. Características físicas e morfológicas de sêmen de touros jovens das raças Gir, Guzerá, Nelore (Bos taurus indicus) e Caracu (Bos taurus taurus). Arq. Bras. Med. Vet. Zootec., v.52, p.461-467, 2000.

SENGER, P.L. Pathways to pregnancy and parturition. 2.ed. Ephrata, Pennsylvania: Cadmus Professional Communications Science Press Division, 2003. 368p.

SILVA, A.E.D.F.; UNANIAN, M.M.; CORDEIRO, C.M.T. et al. Relação da circunferência escrotal e parâmetros da qualidade do sêmen em touros da raça Nelore, PO. Rev. Bras. Zootec., v.31, p.1157-1165, 2002.

TORRES-JÚNIOR， J.R.S.; HENRY， M. Sexual development of Guzerat (Bos taurus indicus) bulls raised in a tropical region. Anim. Reprod., v.2, p.114121, 2005.

VALE FILHO, V.R.; ANDRADE, V.J.; SALVADOR, D.F. et al. Andrological profile and libido of early mature young (one year old) Nelore (Bos taurus indicus) bulls raised under two types of pastures. In: INTERNATIONAL CONGRESS ON ANIMAL REPRODUCTION, 15., 2004, Porto Seguro. Anais... Belo Horizonte: CBRA, 2004. v.1, p.176.

WOLF, F.R.; ALMQUIST, J.O.; HALE, E.B. Prepuberal behavior and puberal characteristics of beef bulls on high nutrient allowance. J. Anim. Sci., v.24, p.761-765, 1965. 